\title{
Application of bivariate mixed counting process models to genetic analysis of rheumatoid arthritis severity Rinku Sutradhar ${ }^{1}$, Dushanthi Pinnaduwage ${ }^{1}$ and Shelley B Bull*1,2
}

\author{
Address: ${ }^{1}$ Samuel Lunenfeld Research Institute of Mount Sinai Hospital, 60 Murray Street, Box \#18, Lebovic Building, 5th Floor, Prosserman \\ Centre, Toronto, Ontario M5T 3L9, Canada and ${ }^{2}$ Department of Public Health Sciences, Faculty of Medicine, University of Toronto, Health \\ Sciences Building, 6th Floor, 155 College Street, Toronto, Ontario MST 3M7, Canada \\ Email: Rinku Sutradhar - rinku.sutradhar@ices.on.ca; Dushanthi Pinnaduwage - pinnad@mshri.on.ca; Shelley B Bull* - bull@mshri.on.ca \\ * Corresponding author
}

from Genetic Analysis Workshop 15

St. Pete Beach, Florida, USA. II-15 November 2006

Published: 18 December 2007

BMC Proceedings 2007, I(SuppI I):SI20

This article is available from: http://www.biomedcentral.com/l753-656I/I/SI/SI 20

(c) 2007 Sutradhar et al; licensee BioMed Central Ltd.

This is an open access article distributed under the terms of the Creative Commons Attribution License (http://creativecommons.org/licenses/by/2.0), which permits unrestricted use, distribution, and reproduction in any medium, provided the original work is properly cited.

\begin{abstract}
We sought to i) identify putative genetic determinants of the severity of rheumatoid arthritis in the NARAC (North American Rheumatoid Arthritis Consortium) data, ii) assess whether known candidate genes for disease status are also associated with disease severity in those affected, and iii) determine whether heterogeneity among the severity phenotypes can be explained by genetic and/or host factors. These questions are addressed by developing bivariate mixed-counting process models for numbers of tender and swollen joints to evaluate genetic association of candidate polymorphisms, such as $D R B I$, and selected single-nucleotide polymorphisms in known candidate genes/regions for rheumatoid arthritis, including PTPN22, and those in the regions identified by a genome-wide linkage scan of disease severity using the dense Illumina single-nucleotide polymorphism panel. The counting process framework provides a flexible approach to account for the duration of rheumatoid arthritis, an attractive feature when modeling severity of a disease. Moreover, we found a gain in efficiency when using a bivariate compared to a univariate counting process model.
\end{abstract}

\section{Background}

The NARAC (North American Rheumatoid Arthritis Consortium) data provided for Genetic Analysis Workshop 15 (GAW15) includes 757 families with 8017 individuals representing multiple ethnicities. The data include information on family relationships, discrete and quantitative phenotypes, covariates, and genome-wide microsatellite genotypes and single-nucleotide polymorphism (SNP) genotypes from Illumina as well as genetic locations for microsatellites and physical locations for SNPs [1].
Our data set for association analysis consists of information on 1492 individuals in 710 families selected from the NAPHENO data. Subjects were included only if information on "TenderJtCt", "SwollenJtCt", "YrOnset", and "YrAscer" are available. The severity phenotypes include the joint count variables "TenderJtCt" and "SwollenJtCt". When examined with respect to the duration of rheumatoid arthritis (RA) (calculated by subtracting "YrOnset" from "YrAscer"), the joint variables can be viewed as a bivariate counting process. Note that the observed time 
since RA onset varied from 1 to 72 years, and the number of joints affected ranged from 0 to 28 and 0 to 26 for tender and swollen, respectively.

Our analytic strategy consisted of the following steps. We began by performing a genome-wide linkage scan using the Illumina SNP panel to identify significant regions of linkage for each count variable. Much of our attention focused on chromosome 6, where a significant region of linkage (harboring the HLA-DRB1 locus) has been previously reported for RA (disease status) [1]. The genomewide linkage analysis not only indicated regions that may be fine-mapped via association analysis, but also suggested differences in linkage signals between the count phenotypes. This motivated the formulation of the bivariate mixed-counting process framework that jointly modeled both tender and swollen processes, as well as detecting differences in patterns of association. We were particularly interested in evaluating the genetic association of candidate polymorphisms, such as DRB1, as well as SNPs selected by genome-wide linkage analysis, or within known candidate genes such as PTPN22, previously reported to be associated with an increased risk of RA [2]. Due to restrictions on available software for linkage analysis, the phenotypes were not modeled in the same manner as for association analysis; the purpose of the linkage analysis was simply to provide a preliminary

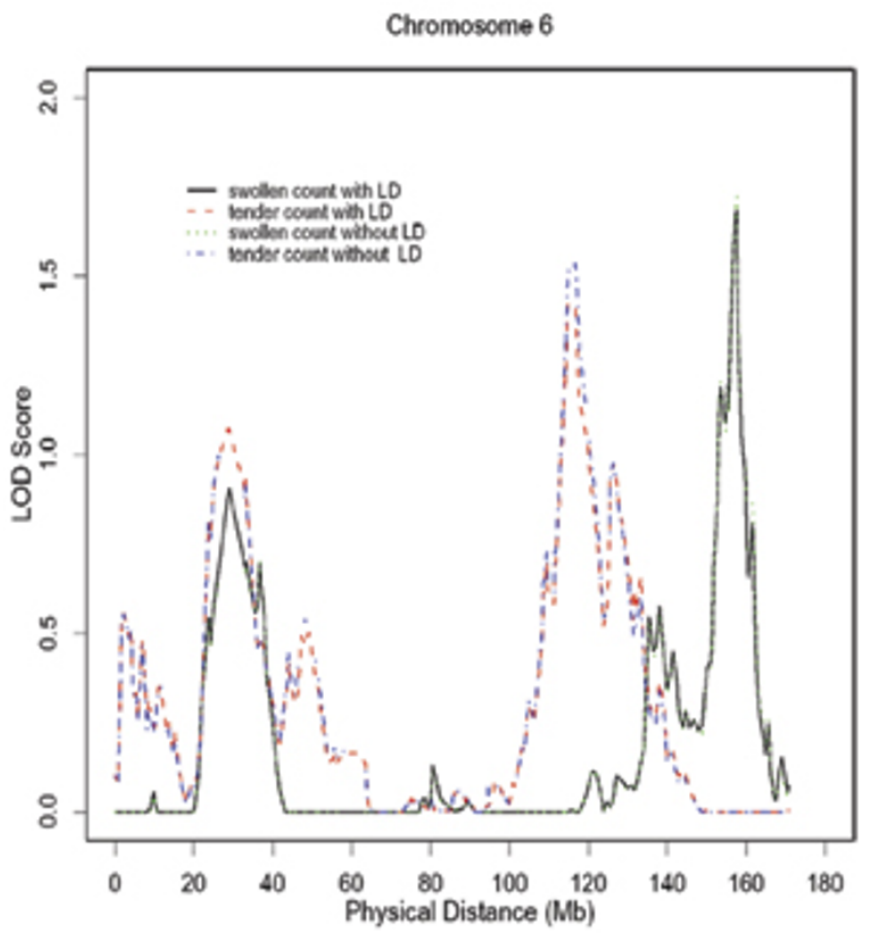

Figure I

LOD score results from genome-wide linkage analysis. understanding of the linkage behavior and potential differences between the tender and swollen joint count phenotypes.

\section{Genome-wide linkage analysis with SNPs}

A genome-wide linkage scan of the count variables available in 1519 individuals in 710 families using 5744 SNPs (Illumina panel) was performed via MERLIN-REGRESS (version 1.0.1) [3], with the square root transformation of the counts treated as quantitative traits. In this regressionbased method [4], we used the sample estimates of the transformed counts as population means and variances, and estimated heritability using the variance-components (VC) option in MERLIN (version 1.0.1) [3], with values of $39 \%$ and $42 \%$ for transformed swollen and tender joint counts, respectively. Because genetic maps were not available for the SNPs, we assumed that $1 \mathrm{Mb}$ is equivalent to $1 \mathrm{cM}$.

Directing our attention to chromosome 6, which has been previously reported to show a significant region of linkage (harboring the HLA-DRB1 locus) for RA, we found two regions of modest signal for each trait (see Fig. 1). One region detected for both count variables spanned 26 to 33 $\mathrm{Mb}$. A second region for the tender count variable spanned 114 to $117 \mathrm{Mb}$, and a differing secondary region for the swollen count variable spanned 155 to $158 \mathrm{Mb}$.
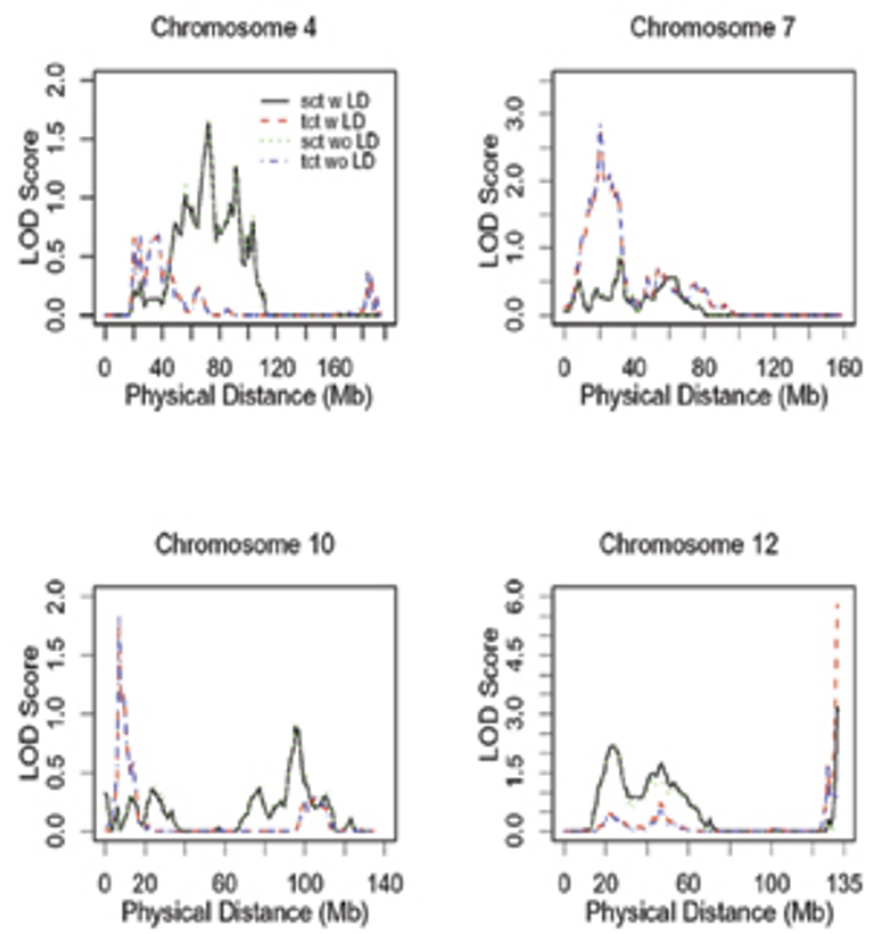
Several other regions of interest were also detected on other chromosomes (Fig. 1). Adjusting this multipoint analysis for linkage disequilibrium using MERLIN produced minor changes (Fig. 1).

\section{Methods \\ A bivariate counting process model for genetic association of $R A$ severity}

Methods for analyzing data on events observed over time have been of considerable interest in recent years. A counting process framework $[5,6]$ models count data, collected at fixed points of ascertainment, by taking duration of disease into consideration (as is the case in a cross-sectional design, provided that the time of disease onset is available). Under the assumption that the numbers of tender and swollen joints do not decrease through time, we have a recurrent event process.

Let $\left\{\mathrm{N}_{i j}(\mathrm{t}), \mathrm{t} \geq 0\right\}$ represent the underlying counting process in which $\mathrm{N}_{i j}(\mathrm{t})$ denotes the number of events experienced by the $j^{\text {th }}$ process of the $i^{\text {th }}$ individual over the continuous time interval $(0, \mathrm{t}]$, where $i=1, \ldots, n$. Note that $j=1,2$ represents the tender and swollen joint counting processes, respectively. Because it is reasonable to specify a model that depends only on the current covariate values rather than on the entire history process in our setting, we formulated a non-homogeneous Poisson process. To handle any substantial inter-individual variation in the model, we introduced a positive individual-specific random effect $u_{i}$ that is shared for both processes of the $i^{\text {th }}$ subject. Conditional on $u_{i}$, the counts of the $i^{\text {th }}$ subject are independent. To be more specific, we assumed that conditional on $u_{i}$, the counting process $\left\{\mathrm{N}_{i j}(\mathrm{t}), \mathrm{t} \geq 0\right\}$ is a nonhomogeneous Poisson process with intensity and mean functions represented as

$$
\lambda_{i j}\left(\mathrm{t} \mid u_{i}\right)=u_{i} \lambda_{0 j}(\mathrm{t}) \exp \left\{\mathbf{x}_{i j}{ }^{\mathrm{T}} \beta_{j}\right\}
$$

and

$$
\Lambda_{i j}\left(\mathrm{t} \mid u_{i}\right)=u_{i} \Lambda_{0 j}(\mathrm{t}) \exp \left\{\mathbf{x}_{i j}^{\mathrm{T}} \beta_{j}\right\},
$$

respectively. The time line is defined as the time since onset of RA. The form of the intensity function is a relative risk or Cox model, with the baseline intensity function for the $j^{\text {th }}$ process $\lambda_{0 j}(\mathrm{t})$ common among all subjects. The covariate vector $\mathbf{x}_{i j}$, specific to the $i^{\text {th }}$ subject, may include candidate polymorphisms (such as DRB1), single SNPs selected through genome-wide linkage scans, or a vector of SNPs chosen to capture variation in a candidate gene or candidate region (such as PTPN22); it may also include characteristics such as sex and smoking history, or any combination of reasonable interactions. The corresponding regression parameter vector $\beta_{j}$ describes the association of genotype with phenotype for the $j^{\text {th }}$ process.
Furthermore, each individual has their own frailty $u_{i}$ acting multiplicatively on the intensity function.

Unless the variance of the underlying mixing distribution is exceptionally large, the gamma random effect provides a robust approach for modeling mixed-Poisson processes [7]. Assuming that $u_{1}, \ldots, u_{n}$ are independent and identically distributed random variables arising from a gamma distribution with mean of 1 and variance of $\phi$, we obtain the expectation $\mathrm{E}\left[\mathrm{N}_{i j}(\mathrm{t})\right]=\mathrm{E}\left(\mathrm{E}\left[\mathrm{N}_{i j}(\mathrm{t}) \mid u_{i}\right]\right)=\Lambda_{0 j}(\mathrm{t})$ $\exp \left\{\mathrm{x}_{i j}{ }^{\mathrm{T}} \beta_{j}\right\}$, which represents the mean number of counts for the $j^{\text {th }}$ process of the $i^{\text {th }}$ individual over the interval $(0$, t]. The parameter $\phi$ is a measure of heterogeneity between individuals that may not have been sufficiently accounted for by the Poisson model alone. Larger values of $\phi$ imply extra-Poisson variation in the model.

We now need to construct a likelihood function based on our bivariate mixed-Poisson process assumptions. Suppose the $i^{\text {th }}$ individual is ascertained at time $\tau_{i^{\prime}}$ which is relative to the time of onset. Conditional on the random effect $u_{i}$, the distribution of the counts $\mathrm{P}\left(\mathrm{N}_{i j}\left(\tau_{i}\right)=\mathrm{n}_{i j} \mid u_{i}\right)$ has a Poisson form. Moreover, under conditional independence, the bivariate distribution of the counts can be computed as $\mathrm{P}\left(\mathrm{n}_{i 1}, \mathrm{n}_{i 2}\right)=\int \mathrm{P}\left(\mathrm{n}_{i 1}, \mathrm{n}_{i 2} \mid u_{i}\right) d \mathrm{G}\left(u_{i}\right)=\int \mathrm{P}\left(\mathrm{n}_{i 1} \mid u_{i}\right)$ $P\left(n_{i 2} \mid u_{i}\right) g\left(u_{i}\right) d u_{i^{\prime}}$ which has a convenient closed form expression due to the gamma-Poisson mixture. Thus the $\log$-likelihood $\log \mathrm{L}(\theta)=\Sigma \log \mathrm{P}\left(\mathrm{n}_{i 1}, \mathrm{n}_{i 2}\right)$ is maximized with respect to $\theta$, which consists of parameters $\beta_{1}, \beta_{2}$, $\phi$, and parameters of the baseline intensity functions. We applied a Newton-Raphson technique, but any non-linear maximization algorithm may be used for this purpose. Furthermore, when maximizing the log-likelihood under the counting process framework, it is most convenient to assume a parametric form such as a Weibull model for the baseline intensity functions, although semi-parametric assumptions using piecewise constant models are also reasonable alternatives.

In the case of a univariate counting process model, there is no joint distribution formulated among the counts for each individual. Rather, the log-likelihood is simply $\mathrm{L}(\theta)$ $=\Sigma \Sigma \log \mathrm{P}\left(\mathrm{n}_{i j}\right)$, where $\mathrm{P}\left(\mathrm{n}_{i j}\right)=\int \mathrm{P}\left(\mathrm{n}_{i j} \mid u_{i}\right) d \mathrm{G}\left(u_{i}\right)=\int \mathrm{P}\left(\mathrm{n}_{i j} \mid u_{i}\right)$ $g\left(u_{i}\right) d u_{i}$.

\section{Results and discussion}

\section{Association analysis of candidate gene DRB I}

We applied our bivariate mixed-counting process model for the severity phenotypes under various covariate models, yielding estimates and standard errors of $\beta_{1}, \beta_{2}$, and $\log \phi$ (Table 1). The asymptotic confidence interval for log $\phi$ indicated a significant amount of extra-Poisson variation in the counts. That is, the number of tender joints varied considerably between patients, as did the number of swollen joints. The heterogeneity appeared to decrease as 
Table I: Results under a bivariate mixed-counting process model for genetic association of RA severity

\begin{tabular}{|c|c|c|c|c|c|c|}
\hline \multirow[b]{2}{*}{ Model } & \multirow[b]{2}{*}{$\log L$} & \multirow[b]{2}{*}{ Heterogeneity log $\phi(\mathrm{SE})$} & \multirow[b]{2}{*}{ Outcome } & \multicolumn{3}{|c|}{ Covariate associations } \\
\hline & & & & $\operatorname{Sex} \beta$ (SE) & Smoking history $\beta$ (SE) & $D R B I \beta$ (SE) \\
\hline No covariates & -10052.06 & $-0.358(0.04)$ & $\begin{array}{l}\text { Tender } \\
\text { Swollen }\end{array}$ & & & \\
\hline \multirow[t]{2}{*}{ With sex } & -10035.23 & $-0.360(0.04)$ & Tender & $-0.185(0.05)$ & & \\
\hline & & & Swollen & $-0.010(0.05)$ & & \\
\hline With sex, & -10031.06 & $-0.365(0.04)$ & Tender & $-0.216(0.06)$ & $0.139(0.04)$ & \\
\hline smoking history & & & Swollen & $-0.035(0.05)$ & $0.110(0.04)$ & \\
\hline With sex, & -10020.33 & $-0.366(0.04)$ & Tender & $-0.216(0.06)$ & $0.139(0.05)$ & $-0.009(0.03)$ \\
\hline smoking history, and DRBI & & & Swollen & $-0.038(0.06)$ & $0.107(0.05)$ & $0.071(0.03)$ \\
\hline
\end{tabular}

more covariates were added to the model, and any remaining variation in the model was captured under this random effects formulation. The log-likelihood increased dramatically as the covariates sex, smoking history, and DRB1 were added to the model. The likelihood ratio (LR) test for the joint contribution of sex to the bivariate model ( 2 degrees of freedom) provided a $p$-value less than $1 \times 10^{-}$ 6 , and the LR test for the joint contribution of DRB1 provided a $p$-value less than 0.0001 . The Wald test detected a significant sex effect for the tender, but not for the swollen joint process. These sex differences were evident in plots of the estimated mean number of counts (Fig. 2). The LR test for the equality of DRB1 between the two counting processes in the bivariate model ( 1 degree of freedom) yielded a $p$-value less than $1 \times 10^{-5}$.

\section{Bivariate Model with no covariates}

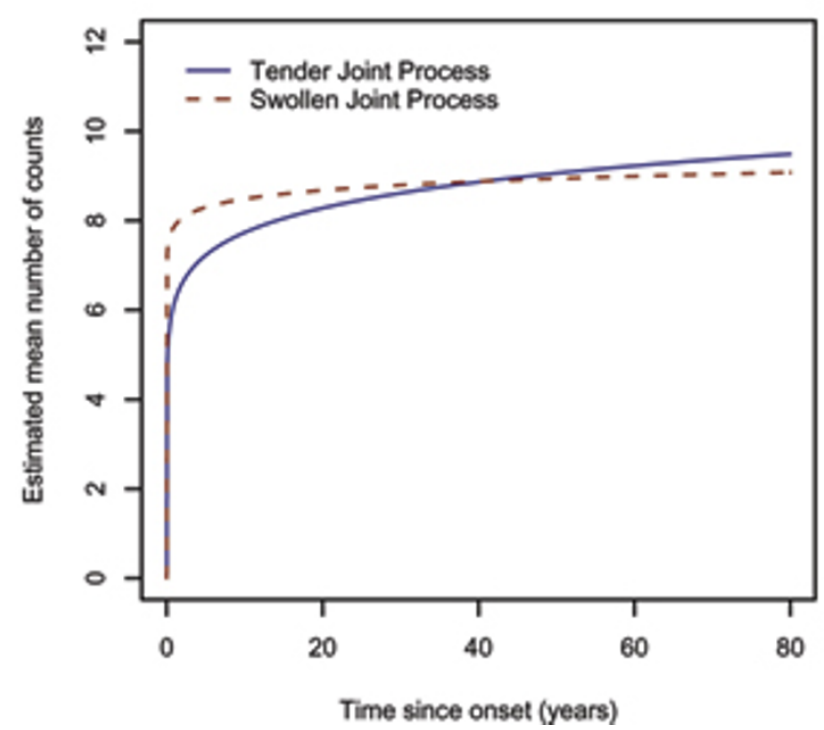

Relative efficiency computations (not shown), obtained by taking the ratio of the variances, suggested a gain in information for the bivariate versus univariate counting process model. For the tender and swollen outcomes, the relative variance ranged from 1.122 to 1.294 and 1.005 to 1.015 , respectively, under various covariates.

\section{Association analysis of the I4 PTPN22 SNPs on} chromosome I and 404 illumina SNPs on chromosome 6 To evaluate the genetic association of candidate gene PTPN22 and SNPs on chromosome 6, we performed various LR tests under both univariate and bivariate mixedcounting process models. Under the univariate model we tested

(i) $\mathrm{H}_{o}: \beta_{\text {tender, } S N P}=0$ and (ii) $\mathrm{H}_{o}: \beta_{\text {swollen, } S N P}=0$,

\section{Bivariate Model with Sex, Smk History, and DRB1}

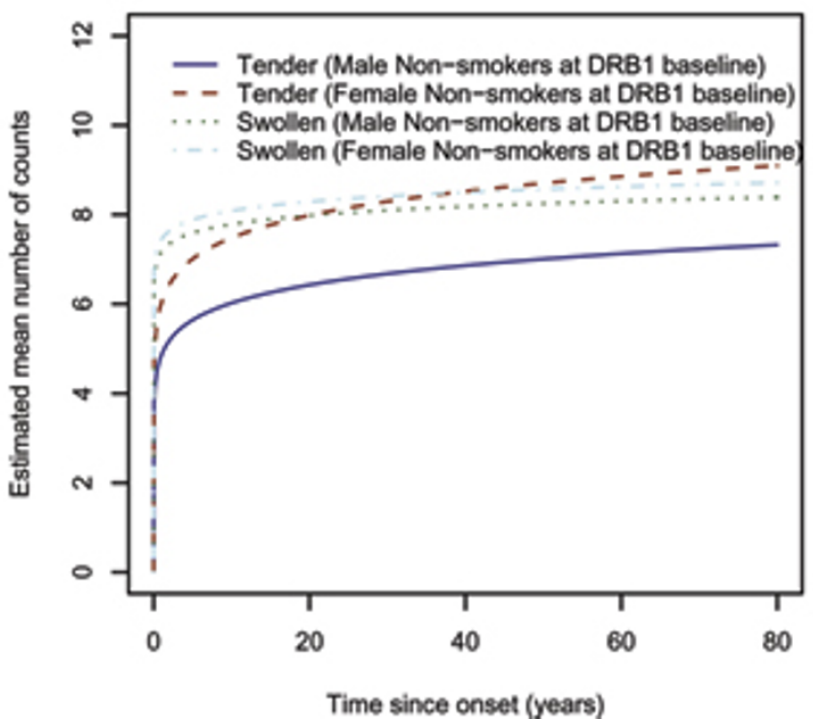

Figure 2

Estimated mean number of counts versus time since RA onset. 
and under the bivariate model we tested

$$
\begin{gathered}
\text { (i) } \mathrm{H}_{o}: \beta_{\text {tender, } S N P}=0, \beta_{\text {swollen, } S N P}=0 \text { and (ii) } \mathrm{H}_{o}: \beta_{\text {tender, } S N P} \\
=\beta_{\text {swollen, SNP. }}
\end{gathered}
$$

Note that, along with sex and smoking history, DRB1 was included in the model since it is significant based on the results of the association analysis above, and also because it has been previously reported to show strong linkage [1] and association with RA. Figure 3 consists of plots of the

\section{Univariate Model for 14 PTPN22 SNPS on Chromosome 1}

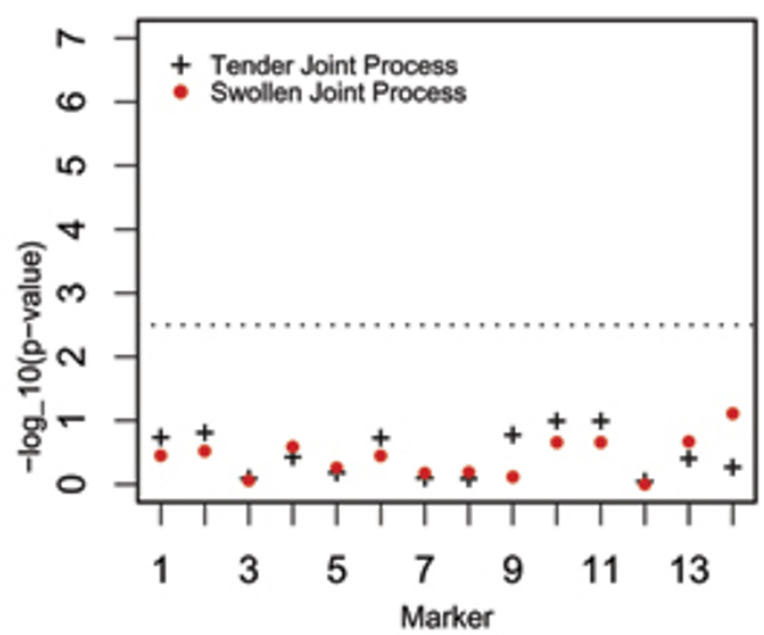

Univariate Model for 404 Illumina SNPs on Chromosome 6

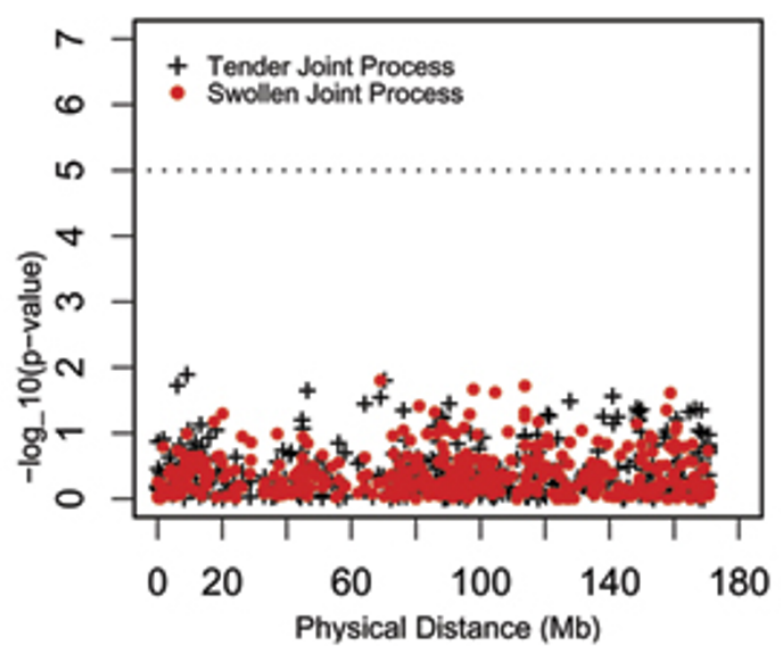

p-values for each SNP. The dashed line indicates a regionwide Bonferroni significance criterion, computed as the ratio of a selected significant $p$-value (0.05) over the number of tests performed.

\section{Conclusion}

The counting process model examines phenotypes in a longitudinal framework by taking duration of disease into account. This model is applicable in a cross-sectional design, provided that the time of disease onset is availa-

\section{Bivariate Model for 14 PTPN22 SNPs on Chromosome 1}

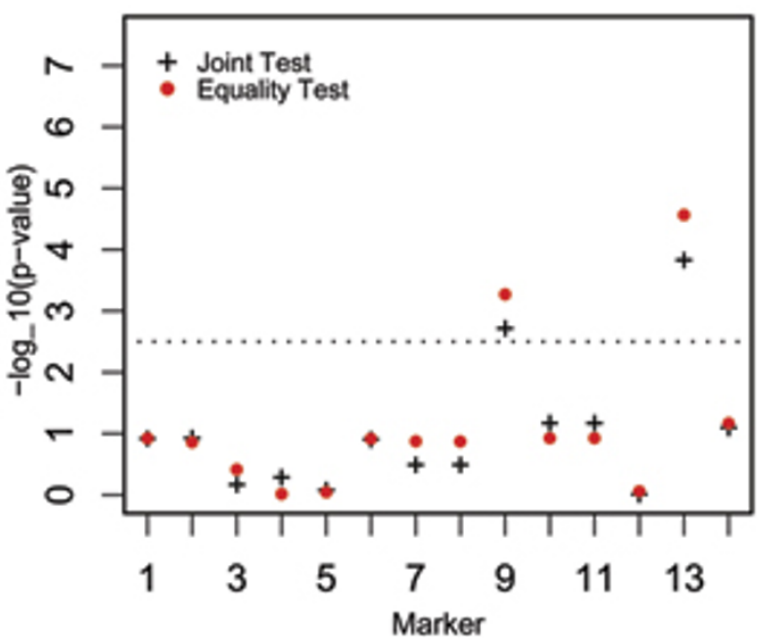

Bivariate Model for 404 Illumina SNPS on Chromosome 6

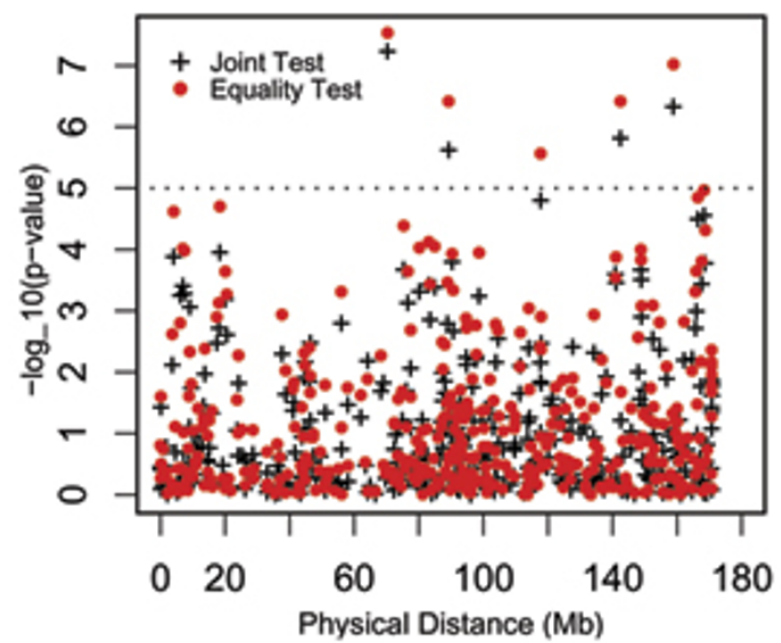

Figure 3

Likelihood ratio test $p$-values under univariate and bivariate models (with sex, smoking history, and DRBI) for SNPs on chromosome I and 6. 
ble. Genetic association analysis under a bivariate model provides a stronger approach than treating each outcome separately. The use of the counting process to model RA severity is novel, so our primary focus is the association between the bivariate count phenotypes and main effects, however an extension to incorporate gene $\times$ gene and gene $x$ environment interactions would be straightforward.

\section{Competing interests}

The author(s) declare that they have no competing interests.

\section{Acknowledgements}

This research was supported by the Canadian Network of Centres of Excellence in Mathematics (MITACS). RS was supported by the Samuel Lunenfeld Research Institute Interface Training Program: Applying Genomics to Human Health (Canadian Institutes of Health Research (CIHR)). SBB held a CIHR Senior Investigator Award.

This article has been published as part of BMC Proceedings Volume I Supplement I, 2007: Genetic Analysis Workshop 15: Gene Expression Analysis and Approaches to Detecting Multiple Functional Loci. The full contents of the supplement are available online at http://www.biomedcentral.com/ $\underline{|753-656| / \mid \text { ? issue=SI. }}$.

\section{References}

I. Amos Cl, Chen WV, Lee A, Li W, Kern M, Lundsten R, Batliwalla F, Wener M, Remmers E, Kastner DA, Criswell LA, Seldin MF, Gregerson PK: High-density SNP analysis of 642 Caucasian families with rheumatoid arthritis identifies two new linkage regions on I Ip I 2 and 2q33. Genes Immun 2006, 7:277-286.

2. Abecasis GR, Cherny SS, Cookson WO, Cardon LR: Merlin-rapid analysis of dense genetic maps using sparse gene flow trees. Nat Genet 2002, 30:97-10I.

3. Carlton VE, Hu X, Chokkalingam AP, Schrodi SJ, Brandon R, Alexander HC, Chang M, Catanese JJ, Leong DU, Ardlie KG, Kastner DL, Seldin MF, Criswell KA, Gregersen PK, Beasley E, Thomson G, Amos $\mathrm{Cl}$, Begovich AB: PTPN22 genetic variation: evidence for multiple variants associated with rheumatoid arthritis. Am J Hum Genet 2005, 77:567-58I.

4. Sham PC, Purcell S, Cherny SS, Abecasis GR: Powerful regressionbased quantitative-trait linkage analysis of general pedigrees. Am J Hum Genet 2002, 7 I:238-253.

5. Andersen PK, Borgan O, Gill RD, Keiding N: Statistical Models Based on Counting Processes New York: Springer-Verlag; 1993.

6. Lawless JF: Statistical Models and Methods for Lifetime Data 2nd edition. Hoboken: John Wiley \& Sons; 2003.

7. Cook RJ, $\mathrm{Ng}$ ETM: Adjusted score tests of homogeneity for Poisson processes. J Am Stat Assoc 1999, 94:308-319.

\section{Publish with Biomed Central and every scientist can read your work free of charge}

"BioMed Central will be the most significant development for disseminating the results of biomedical research in our lifetime. "

Sir Paul Nurse, Cancer Research UK

Your research papers will be:

- available free of charge to the entire biomedical community

- peer reviewed and published immediately upon acceptance

- cited in PubMed and archived on PubMed Central

- yours - you keep the copyright

Submit your manuscript here:

http://www.biomedcentral.com/info/publishing_adv.asp
BioMedcentral 